\title{
A synthetic exponentially weighted moving average control chart to monitor process median based on ranked set sampling
}

\author{
Jing Wen $\mathrm{Ng}^{1 *}$, Voon Hee Wong ${ }^{1}$, and Sook Theng Pang ${ }^{1}$ \\ ${ }^{1}$ Department of Mathematical and Actuarial Sciences, Lee Kong Chian Faculty of Engineering and \\ Science, Universiti Tunku Abdul Rahman, 43000 Kajang, Selangor, Malaysia
}

\begin{abstract}
Exponentially Weighted Moving Average (EWMA) control charts yield insights into data in a way more comprehensible to the practitioners and researchers because of its capability in discovering small to moderate process mean shifts. EWMA control chart is incorporated with conforming run length (CRL) chart, named synthetic EWMA chart, to enhance the performance of the chart in detecting the out-of-control signal. Synthetic EWMA chart based on ranked set sampling (RSS) for monitoring process mean has been proposed as it advanced the detection of chart over a series of mean shifts. With the situation that normality assumption is scarcely attain in practice, we proposed synthetic EWMA median chart based on RSS. Rather than select average run length (ARL) as sole performance evaluating tool, the median and percentiles of run-length distribution are used to examine the performance of the proposed chart as it provides more information on the entire run-length distribution. Nearoptimal parameters of the proposed chart will be acquired by setting the incontrol ARL at a designated value. The run length performances of the proposed chart are then compared with the existing charts such as EWMA median chart based on RSS.
\end{abstract}

\section{Introduction}

To ensure the sustainability and the improvements of the quality of the products over time and to assess the effectiveness of a manufacturing process, Statistical Process Control (SPC) techniques are introduced by a physicist, Dr Walter Andrew Shewhart, to accomplish the objective [1]. SPC techniques incorporate basic statistical concept into mathematical formulae and graphical presentation that can be utilized by non-statisticians to observe the change in process and trends in the past [2]. Control chart is a popular and valuable tool in SPC as it is a simple graph used to inspect the current data by using historical control limits which leads to inferences about whether the process variation is consistent or random.

The modern advanced statistical quality monitoring techniques such as exponentially weighted moving average (EWMA) control chart comes from the solid concept of the control chart. By comparing the advanced chart with the traditional Shewhart control chart, the

*Corresponding author: ngjw@utar.edu.my 
sensitivity of the advanced chart to small process mean shift is higher but the sensitivity to a larger shift in a process mean is lower than the traditional control chart. To overcome the weakness of the advanced chart, a further research has been put forward by Scariano and Calzada [3]. A generalized synthetic control chart is introduced by incorporating EWMA control charts with the conforming run length (CRL) control chart. These charts are given the name of Synthetic EWMA control chart. The new chart overtakes the optimal EWMA control chart in terms of its efficiency to identify an out-of-control event. The idea of conforming run length (CRL) control chart has been presented by Bourke [4] to identify the status of the process. Thus, the CRL value is the number of the samples that have been captured before the first test statistic lies outside the control limit or since the last test statistic that lies outside the control limits. The value will then be used to compare with a threshold, $L$ to determine the status of the process and corrective action will be taken. The $L$ is normally defined by the user as a benchmark to check the fraction nonconforming of the samples data. This is dissimilar with the traditional control chart as an uncontrollable signal would not be created directly when sample mean falls outside the control limits. Commonly, synthetic chart transcends the traditional control chart when optimal synthetic chart is chosen to keep track of a mean shift within a certain range.

Lately, the classical ranked set sampling (RSS) is renowned in the creation of upgraded control charts. The mathematical foundation of RSS scheme have been suggested by Takahasi and Wakimoto [5] and they proved that the sample mean acquired based on RSS is unbiased and superior than the one obtained based on the traditional simple random sampling (SRS) method. There is likelihood occurrence of ranking faults. However, Dell and Clutter [6] verified that the estimator of the population mean based on RSS is still dominate the SRS mean estimator except ranking is worse than random ordering. Based on these evidence, some researchers have implemented the use of RSS scheme in control chart; for instance, Salazar and Sinha [7] implemented the scheme into the classical Shewhart control chart to examine the process mean; Muttlak and Al-Sabah [8] had proposed a new control chart based on perfect and imperfect RSS schemes to boost up the sensitivity of a control chart. Moreover, Abdul Haq et al. [9] introduced a new Synthetic EWMA control charts based on RSS and MRSS and showed that the proposed charts overtake existing charts based on SRS.

Currently, median chart $(\tilde{X})$ has been widely chosen as a replacement to the traditional mean $(\bar{X})$ chart due to its capability to recognise small variation from normality assumption. Commonly, $\bar{X}$ chart is selected to identify large mean shift in a process whereas EWMA $\bar{X}$ chart is always recognized in monitoring small to moderate mean shifts in a process. However, when the dataset is contaminated and contain outliers, Human et al. [10] pointed out that EWMA $\bar{X}$ chart is not appropriate to use to evaluate the data as the inferences obtained from the chart under this situation will be untrustworthy. Based on Tan and Chong

[11], one of the strength of $\tilde{X}$ chart is that it can be used to keep track an ongoing process

running for some times. In addition, EWMA $\tilde{X}$ chart with estimated parameters has been established by Castagliola, Maravelakis, and Figueiredo [12] to replace EWMA $\bar{X}$ chart and the new chart parameters had been proposed to minimize the difference between the known and estimated parameters cases.

Average run length (ARL) is defined as the expected number of subgroups obtained until an out-of-control event is captured by a control chart. The ARL criterion is commonly selected as a method to study the efficiency of various control charts. However, the focus on the presentation of ARL as an individual performance evaluation tool has been raising concern and criticised by many researchers. From Khoo and Wong [13], the run-length 
distribution is observed to be greatly skewed to the right for the in control or slightly out of control process. Therefore, when the size of the process mean shift changes, the run-length distribution will vary in skewness accordingly. Some of the crucial information on the run length distribution might be overlooked. A better performance evaluation substitute for the ARL has been proposed by Zhou et al. [14], which is the percentiles of the run-length distribution, as it can contribute more detailed understanding on the entire spread of the distribution. For instance, practitioners are assured that the charts will signal an alarm by the $200^{\text {th }}$ sample at least $50 \%$ of the time when the $50^{\text {th }}$ run length percentile is equal to 200 . Besides that, when the $10^{\text {th }}$ run length percentile is equal to 25 , practitioners are $90 \%$ confident that a false signal does not take place within the first 25 samples. Additionally, the difference between the $5^{\text {th }}$ and $95^{\text {th }}$ run length percentiles provides the data on the disparity of the distribution.

In this paper, we extend the work of Abdul Haq et al. [9] and propose a Synthetic EWMA median chart based on RSS scheme for a quicker discovery of small to large mean shifts. The near-optimal chart's parameters are figured by setting the in-control ARL value to be nearly equal to 370 . Then, we investigate the efficiency of the proposed chart in determining an outof-control event by examine the run length percentiles of the chart. Lastly, the proposed chart is further compared with the existing charts such as EWMA median chart based on RSS scheme in terms of MRL to study the superiority of the chart.

\section{Ranked set sampling scheme}

In this section, the procedure to implement RSS schemes based on perfect rankings is explained as follows:

Step 1 . Begin the process by generating $n$ random sets from the population, each of size $n$ units.

Step 2. Rank the units within each set with respect to a variable of interest.

Step 3. Take an actual measurement from the unit with the $1^{\text {st }}$ smallest rank from the $1^{\text {st }}$ set. Then, take an actual measurement from the unit with the $2^{\text {nd }}$ smallest rank from the $2^{\text {nd }}$ set. Continue the procedure for the $c^{\text {th }}$ smallest ranked unit from the $c^{\text {th }}$ set, where $c=3,4, \ldots, n$ .In the end of this step, we acquire a sample of size $n$.

Step 4. Repeat Step 1 to Step $3 m$ times until a complete cycle of data, $r=n m$ has been obtained. These $n m$ units form the RSS data.

Figuratively, throughout this paper, let $X_{a b j}$ to be standard normal random variables, where, $a=1,2, \ldots, n, b=1,2, \ldots, n$ and $j=1,2, \ldots, m$.Now we let sequence of samples at the cycle $j$,

$$
S_{j}=\left(X_{11 j}, X_{12 j}, \ldots, X_{1 n j}\right),\left(X_{21 j}, X_{22 j}, \ldots, X_{2 n j}\right), \ldots,\left(X_{n 1 j}, X_{n 2 j}, \ldots, X_{n n j}\right)
$$

where each of the $\left(X_{a 1 j}, X_{a 2 j}, \ldots, X_{a n j}\right)$ is a simple random sample. Then, the RSS scheme procedure is applied on the sequence of samples at the cycle $j, S_{j}$ to obtain a ranked set sample of size $n$, symbolised by $X_{a(b: n) j}$, where $X_{a(b: n) j}$ is the $a^{\text {th }}$ order statistics of the sample $\left(X_{a 1 j}, X_{a 2 j}, \ldots, X_{a n j}\right)$. The procedure to acquire the first set of RSS data in the first cycle is presented on Fig. 1. 


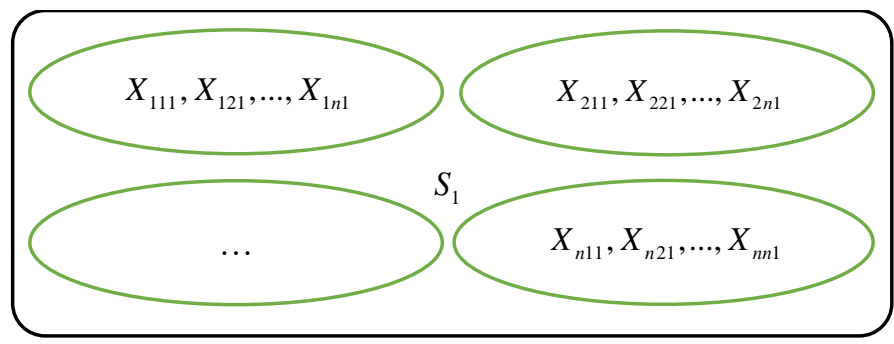

Apply the RSS scheme on this sequence of sample to obtain a ranked set sample of size $n$ for the first cycle

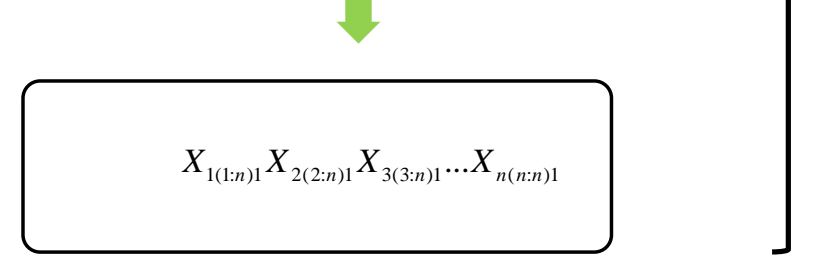

Repeat $m$ times to obtain $m$ cycles of a ranked set sample with total sample size $r=n m$.

Fig. 1. The procedure to obtain the first set of RSS data in the first cycle.

\section{Proposed synthetic EWMA median chart}

The sample mean and the variance of a ranked set sample presented by Takahasi and Wakimoto [5] are commonly implemented for the creation of mean chart. Some slight alteration on the computation of the sample mean and variance for the median chart is required as it is associated to order statistics. The sample mean for Synthetic EWMA median chart is

$$
\bar{X}_{\mathrm{RSS}}=\frac{1}{m} \sum_{j=1}^{m} \tilde{X}_{a j}
$$

where $\tilde{X}_{a j}$ is the sample median of the subgroup $a$, and sample variance estimator for the Synthetic EWMA median chart is

$$
\operatorname{Var}\left(\bar{X}_{\mathrm{RSS}}\right)=\operatorname{Var}\left(\bar{X}_{\mathrm{SRS}}\right)-\frac{1}{n^{2}} \sum_{b=1}^{n}\left(\tilde{X}_{(b: n) j}-\bar{X}_{\tilde{X}_{(b n n) j}}\right)^{2}
$$

where $\tilde{X}_{(b: n) j}$ is the median of the $b^{\text {th }}$ order statistics and $\bar{X}_{\tilde{X}_{(b n) j}}$ is the mean of the median of the $b^{\text {th }}$ order statistics. For notational convenience, let $\bar{X}_{\mathrm{RSS}}=\mu_{X, \mathrm{RSS}}$ and $\operatorname{Var}\left(\bar{X}_{\mathrm{RSS}}\right)=\sigma_{X, \mathrm{RSS}}^{2}$.

The Synthetic EWMA median chart based on perfect RSS schemes is constructed as follows:

Step 1. A ranked set sample $\left\{X_{1(1: n) j}, X_{2(2: n) j}, \ldots, X_{n(n: n) j}\right\}$ of $n$ independent normal random variables is obtained, where $j$ is the subgroup number. Then $\left\{X_{1(1: n) j,(1)}, \ldots, X_{n(n: n) j,(n)}\right\}$ is the $j^{\text {th }}$ 
subgroup organised in ascending order. The sample median of subgroup $j$ is

$$
\tilde{X}_{j}=\left\{\begin{array}{cl}
X_{j,\left(\frac{n+1}{2}\right)} & \text { if } n \text { is odd. } \\
\frac{X_{j,\left(\frac{n}{2}\right)}+X_{j,\left(\frac{n}{2}+1\right)}}{2}, & \text { if } n \text { is even. }
\end{array}\right.
$$

In this paper, we are generally focusing on the odd sample size cases only.

Step 2. The mean and standard deviation of the samples are calculated to form the control limits of the proposed chart,

$$
\mathrm{UCL}=\mu_{0, \mathrm{RSS}}+K \sigma_{0, \mathrm{RSS}}
$$

and

$$
\mathrm{LCL}=\mu_{0, \mathrm{RSS}}-K \sigma_{0, \mathrm{RSS}}
$$

where UCL denotes the upper control limit, LCL denotes the lower control limit, $\mu_{0, \mathrm{RSS}}$ and $\sigma_{0, \mathrm{RSS}}$ represents the in-control RSS mean and standard deviation correspondingly, $K>0$ is a constant to be discovered such that the in-control ARL of the EWMA control chart is roughly equal to 370 .

Step 3. The EWMA sequence based on $\tilde{X}_{j}$, for $j=1,2, \ldots$ is computed as

$$
Z_{j, \mathrm{RSS}}=(1-\lambda) Z_{j-1, \mathrm{RSS}}+\lambda \tilde{X}_{j}
$$

where $0<\lambda \leq 1$ is the smoothing constant and $Z_{0, \mathrm{RsS}}=\mu_{0, \mathrm{RSS}}$.

Step 4. $Z_{j, \mathrm{RSS}}$ is plotted with the control limits against time, $j$. If $Z_{j, \mathrm{RSS}}$ is situated between the control limits, in which $\mathrm{LCL}<Z_{j, \mathrm{Rss}} \leq \mathrm{UCL}$, the process is considered to be in control and the procedure returns to Step 3; If at time $j, Z_{j, \mathrm{RSS}}$ lies outside the control limits, in which $Z_{j, \mathrm{RSS}}>\mathrm{UCL}$ or $Z_{j, \mathrm{RSS}}<\mathrm{LCL}$, a sample CRL value can be acquired by considering that test statistic a nonconforming sample and sum up the number of samples that have been taken between this and the former nonconforming sample. If the sample CRL value is larger than the user defined lower limit $L$, the construction of the EWMA control chart is recommenced by setting $Z_{j, \text { RSS }}=\mu_{0, \mathrm{RSS}}$ and the procedure is returned to Step 3 . However, if the sample CRL is smaller than $L$, the EWMA chart generates an out-of-control signal and the procedure continues to Step 5.

Step 5. The process is terminated, and corrective action is taken to examine the out-of-control signal and remove the assignable causes.

Step 6. Step 1 to Step 5 is simulated for 100000 times to estimate the percentiles of runlength distribution of the proposed chart.

The control chart structure for Synthetic EWMA median chart based on RSS scheme is complex and it is challenging to find the particular probability distribution of $\tilde{X}_{j, \text { Rss }}$ under RSS scheme. Therefore, the optimization procedures are difficult to apply on our proposed 
Table 1. Dictionary of the value $K$ based on the desired parameters, $\lambda$ and $L$ of proposed chart based on ranked set sampling (RSS) scheme.

\begin{tabular}{|l|l|l|l|l|}
\hline & \multicolumn{5}{|c|}{$L$} \\
\hline$\lambda$ & 1 & 10 & 20 & 50 \\
\hline 0.05 & 0.21 & 0.45 & 0.52 & 0.60 \\
\hline 0.25 & 1.02 & 1.55 & 1.66 & 1.80 \\
\hline 0.50 & 1.93 & 2.56 & 2.70 & 2.88 \\
\hline 0.75 & 2.81 & 3.51 & 3.69 & 3.92 \\
\hline
\end{tabular}

Table 2. Percentiles of the run-length distribution for the synthetic EWMA median chart based on RSS scheme when $n=5, \delta=0$ for $L=1,10,20,50$.

\begin{tabular}{|c|c|c|c|c|c|c|c|c|c|c|c|c|c|c|}
\hline & & \multicolumn{13}{|c|}{ Percentiles of Run Length Distribution } \\
\hline$\lambda$ & $L$ & $5^{\text {th }}$ & 10th & 20th & $25^{\text {th }}$ & 30th & 40th & 50 th & 60 th & 70th & 75th & 80th & 90th & 95th \\
\hline \multirow[t]{3}{*}{0.05} & 1 & 12 & 33 & 77.5 & 103 & 128 & 195 & 256 & 333 & 444 & 512 & 597 & 866 & 1128.5 \\
\hline & 10 & 7 & 9 & 54 & 79 & 107 & 169 & 244 & 337.5 & 452 & 522 & 613 & 890 & 1165 \\
\hline & 50 & 14 & 20 & 33 & 40 & 47 & 134 & 217 & 315 & 448 & 535 & 635 & 947.5 & 1276 \\
\hline \multirow[t]{3}{*}{0.25} & 1 & 9 & 30 & 74 & 98 & 126 & 183 & 253.5 & 337 & 448.5 & 523 & 608 & 881 & 1129.5 \\
\hline & 10 & 5 & 8 & 42 & 69 & 100 & 161 & 236 & 323 & 443 & 516.5 & 618 & 914 & 1227 \\
\hline & 50 & 9 & 16 & 30 & 38 & 46 & 127 & 209.5 & 316 & 447 & 530 & 636 & 961 & 1297 \\
\hline \multirow[t]{4}{*}{0.50} & 1 & 6 & 28 & 74 & 99 & 124 & 182 & 250 & 336 & 451.5 & 522 & 609 & 878 & 1133.5 \\
\hline & 10 & 4 & 7 & 38 & 67 & 97 & 160 & 239 & 335 & 454 & 529.5 & 625.5 & 906 & 1196 \\
\hline & 20 & 6 & 10 & 19 & 52.5 & 83 & 149 & 226 & 326.5 & 460 & 537 & 631 & 919 & 1224 \\
\hline & 50 & 8 & 14 & 29 & 37 & 45 & 123.5 & 210 & 318 & 451 & 535 & 644 & 961 & 1294 \\
\hline \multirow[t]{3}{*}{0.75} & 1 & 1 & 22 & 67 & 91 & 119 & 181 & 250 & 338 & 445.5 & 519 & 607 & 874 & 1138 \\
\hline & 10 & 4 & 7 & 40 & 66 & 95 & 159 & 236 & 331.5 & 450 & 528 & 630 & 917 & 1199 \\
\hline & 20 & 4 & 9 & 19 & 47 & 80 & 146 & 223 & 322 & 455 & 536 & 633 & 928.5 & 1269 \\
\hline
\end{tabular}


Table 3. ARLs, SDRLs, and MRLs for the proposed control chart when $\lambda=\{0.05,0.25,0.50,0.75\}, \delta \in\{0.00,0.25,0.50,0.75,1.00,1.50,2.00\}$ and $L=1,10,20,50$.

\begin{tabular}{|c|c|c|c|c|c|c|c|c|c|c|c|c|c|}
\hline & & \multicolumn{3}{|c|}{$\lambda=0.05$} & \multicolumn{3}{|c|}{$\lambda=0.25$} & \multicolumn{3}{|c|}{$\lambda=0.50$} & \multicolumn{3}{|c|}{$\lambda=0.75$} \\
\hline$\lambda$ & $L$ & ARL & SDRL & MRL & ARL & SDRL & MRL & ARL & SDRL & MRL & ARL & SDRL & MRL \\
\hline \multirow[t]{3}{*}{0.00} & 1 & 370.76 & 381.87 & 256 & 370.12 & 375.05 & 253.5 & 370.22 & 381.15 & 250 & 370.41 & 392.35 & 250 \\
\hline & 10 & 370.17 & 403.55 & 244 & 370.42 & 420.09 & 236 & 370.70 & 414.58 & 239 & 370.09 & 419.94 & 236 \\
\hline & 50 & 370.71 & 444.99 & 217 & 370.50 & 450.09 & 209.5 & 370.50 & 453.99 & 210 & 370.36 & 467.31 & 199 \\
\hline \multirow[t]{4}{*}{0.25} & 1 & 298.08 & 303.11 & 206 & 295.02 & 300.83 & 202 & 277.05 & 288.21 & 189.5 & 299.27 & 312.50 & 199 \\
\hline & 10 & 143.84 & 157.32 & 93 & 179.65 & 206.29 & 111 & 221.64 & 253.50 & 140 & 257.68 & 293.87 & 163 \\
\hline & 20 & 111.21 & 127.47 & 67 & 156.73 & 191.91 & 89 & 212.25 & 257.50 & 125.5 & 249.41 & 301.70 & 143 \\
\hline & 50 & 82.36 & 99.37 & 38 & 141.59 & 180.58 & 55 & 197.52 & 253.70 & 96 & 246.06 & 312.13 & 131 \\
\hline \multirow[t]{4}{*}{0.50} & 1 & 172.61 & 176.88 & 119 & 156.62 & 163.18 & 106 & 158.26 & 164.40 & 107 & 175.50 & 184.56 & 118 \\
\hline & 10 & 40.12 & 44.22 & 24 & 52.57 & 62.77 & 30 & 77.93 & 92.76 & 45 & 116.08 & 139.25 & 68 \\
\hline & 20 & 28.14 & 29.59 & 15 & 40.89 & 50.97 & 17 & 70.29 & 89.33 & 35 & 106.34 & 132.75 & 57 \\
\hline & 50 & 24.57 & 19.98 & 19 & 37.16 & 43.83 & 22 & 64.61 & 81.16 & 32 & 104.69 & 136.28 & 42 \\
\hline \multirow[t]{3}{*}{0.75} & 1 & 89.17 & 90.37 & 62 & 81.04 & 83.64 & 55 & 79.55 & 85.14 & 52 & 90.19 & 97.47 & 60 \\
\hline & 10 & 15.65 & 15.49 & 9 & 19.30 & 21.95 & 9 & 29.70 & 36.52 & 14 & 46.27 & 58.17 & 24 \\
\hline & 20 & 13.05 & 10.09 & 10 & 15.93 & 17.23 & 10 & 25.79 & 31.46 & 14 & 42.65 & 54.30 & 17 \\
\hline \multirow[t]{4}{*}{1.00} & 1 & 47.11 & 49.31 & 32 & 42.44 & 44.85 & 28 & 41.27 & 43.80 & 27.5 & 45.32 & 50.65 & 29 \\
\hline & 10 & 8.84 & 7.06 & 7 & 9.42 & 9.45 & 6 & 13.48 & 15.98 & 7 & 21.42 & 27.68 & 9 \\
\hline & 20 & 8.72 & 4.62 & 8 & 8.92 & 7.25 & 7 & 12.45 & 13.94 & 8 & 19.73 & 24.66 & 11 \\
\hline & 50 & 10.11 & 4.55 & 9 & 10.05 & 7.05 & 8 & 13.57 & 12.76 & 10 & 21.55 & 24.28 & 14 \\
\hline \multirow[t]{4}{*}{1.50} & 1 & 16.74 & 17.59 & 11 & 14.87 & 15.51 & 10 & 13.54 & 14.61 & 9 & 14.36 & 16.09 & 9 \\
\hline & 10 & 4.91 & 2.15 & 5 & 4.47 & 2.81 & 4 & 5.06 & 4.48 & 4 & 6.61 & 7.28 & 4 \\
\hline & 20 & 5.53 & 2.01 & 5 & 4.81 & 2.63 & 4 & 5.29 & 3.85 & 4 & 6.87 & 6.54 & 5 \\
\hline & 50 & 6.38 & 2.24 & 6 & 5.37 & 2.90 & 5 & 6.21 & 4.61 & 5 & 8.39 & 7.44 & 6 \\
\hline \multirow[t]{4}{*}{2.00} & 1 & 7.34 & 7.54 & 5 & 6.44 & 6.62 & 4 & 5.76 & 6.17 & 4 & 5.93 & 6.56 & 3 \\
\hline & 10 & 3.64 & 1.21 & 3 & 3.07 & 1.39 & 3 & 3.02 & 1.85 & 3 & 3.40 & 2.71 & 3 \\
\hline & 20 & 4.16 & 1.28 & 4 & 3.30 & 1.43 & 3 & 3.29 & 1.95 & 3 & 3.69 & 2.82 & 3 \\
\hline & 50 & 4.74 & 1.41 & 5 & 3.61 & 1.59 & 3 & 3.68 & 2.20 & 3 & 4.41 & 3.41 & 3 \\
\hline
\end{tabular}


chart. In order to partly overcome the problematic circumstances, the near-optimal Synthetic EWMA median chart based on RSS scheme is constructed by using different selections of parameters values in Table 1 that give an in-control ARL of 370 with the fixed sample size, $n$, and size of mean shift, $\delta$. SAS is used to simulate the parameter involved in the Synthetic EWMA-RSS median chart, $K$.

In the construction of the proposed chart, $n$ is chosen to be $5, L \in\{1,10,20,50\}$, and $\lambda \in\{0.05,0.25,0.50,0.75\}$. Based on the settings, the near-optimal parameters $K$ are attained and listed in Table 1.

\section{The performance of the synthetic EWMA median chart}

After the optimal parameter $K$ values are acquired, the SAS programming language is used to simulate a set of data to assess the performance of the proposed chart. In this study, different values of $\lambda, L$ and $K$ are considered, and 100,000 replications is generated to calculate the ARL, standard deviation of run length (SDRL), median run length (MRL) and percentiles of the run length distributions. The performance outcomes of the proposed chart when there is no mean shift in the process are presented in Table 2. Besides that, in this study, for the out-of-control process, only $\mu+\delta \sigma$ are measured. A set of mean shifts, $\delta \in\{0.25,0.50,0.75,1.00,1.50,2.00\}$ is considered for an out-of-control process and the result is displayed in Table 3 .

From Table 2, when an in-control process or $\delta=0$ is measured, it is clearly shown the ARL value of 370 is situated between $60^{\text {th }}$ and $70^{\text {th }}$ run-length percentiles. As the median is less than mean, the shape of distribution of the proposed chart is skewed to the right. For instance, in Table 2, when the process is in-control with $L=1$ and $\lambda=0.25$, it can be noticed that the ARL value is 370.12 and it is situated between the $60^{\text {th }}$ and $70^{\text {th }}$ run-length percentiles. Conversely, the value of the $50^{\text {th }}$ run-length percentiles or Median Run Length (MRL) is 253.5. The problem can be further illustrated from an example taken in Table 3 . When the $L=10$ and $\lambda=0.05$ and $\delta=0.50$, the ARL value is 40.12 and the MRL value is 24 . In this case, the practitioners may be misleadingly deduced that the process will produce an uncontrollable signal by $40^{\text {th }}$ sample with a likelihood of $50 \%$. However, the out-of-control process occurs by $24^{\text {th }}$ sample, which is quicker than indicated. The ARL value represents the mean of subgroups that are registered on a control chart before an out-of-control event is signalled but not the likelihood of getting it. Hence, the chart's performance may be wrongly analysed if the analysis is prepared based on the ARL value alone. However, the ARL value is closer to MRL value when the mean shift, $\delta$ rises. This can be demonstrated from an instance in Table 3 when the $L=50, \lambda=0.50$ and $\delta=2$. The median value is noticed to be approximately equal to the mean value because the profile of the distribution shifts to almost symmetric for an out-of-control process. It can be clearly observed that the amount of skew of the distribution has greater impacts on the ARL value than MRL value.

Additionally, the 5th and 95th run-length values provide the key to acquire some vital knowledges on the spread and variation of the run-length distribution. For example, by looking at the case of $L=1, \lambda=0.50, \delta=0$ in Table 2 , the 5 th and 95 th run-length values of proposed chart are 6 and 1133.5 respectively. To determine the variation of the distribution for the proposed chart, we can use 1133.5 to subtract by 6 . This means that spread of runlength distribution of proposed chart is vary by 1127.5 .

Furthermore, an early incorrect signal can be simply noticed from the assessment of the lower run-length percentile. For example, in Table 2, when there is no mean shift in the process, $L=50$ and $\lambda=0.75$, there is still a $5 \%$ possibility that an incorrect alarm will take place 
by the 6th sample. Besides that, from Table 2, the in-control ARL values are considerably larger than the lower percentile values. These features indicate the possibility that a vast number of false signals will be produced when the process monitoring begins.

Table 4. ARLs for the EWMA median chart based on RSS scheme when $\delta \in\{0.00,0.25,0.50,0.75,1.00,1.50,2.00\}, n=5$ and $\lambda=0.05,0.25,0.50,0.75$.

\begin{tabular}{|l|l|l|l|l|}
\hline$\delta$ & $\lambda=0.05$ & $\lambda=0.25$ & $\lambda=0.50$ & $\lambda=0.75$ \\
\hline 0.00 & 370.73 & 370.19 & 370.73 & 370.81 \\
\hline 0.25 & 94.54 & 175.96 & 230.70 & 276.58 \\
\hline 0.50 & 34.35 & 59.09 & 102.55 & 144.98 \\
\hline 0.75 & 19.55 & 26.39 & 46.40 & 76.51 \\
\hline 1.00 & 13.30 & 14.69 & 24.10 & 41.58 \\
\hline 1.50 & 8.29 & 6.97 & 9.10 & 14.56 \\
\hline 2.00 & 6.02 & 4.45 & 4.81 & 6.81 \\
\hline
\end{tabular}

Table 5. SDRLs for the EWMA median chart based on RSS scheme when $\delta \in\{0.00,0.25,0.50,0.75,1.00,1.50,2.00\}, n=5$ and $\lambda=0.05,0.25,0.50,0.75$.

\begin{tabular}{|l|l|l|l|l|}
\hline$\delta$ & $\lambda=0.05$ & $\lambda=0.25$ & $\lambda=0.50$ & $\lambda=0.75$ \\
\hline 0.00 & 258.73 & 369.22 & 378.37 & 371.49 \\
\hline 0.25 & 77.94 & 173.32 & 228.66 & 173.63 \\
\hline 0.50 & 21.80 & 55.52 & 101.25 & 146.24 \\
\hline 0.75 & 9.94 & 22.17 & 44.69 & 75.20 \\
\hline 1.00 & 5.60 & 10.93 & 22.04 & 40.58 \\
\hline 1.50 & 2.72 & 3.96 & 7.14 & 13.38 \\
\hline 2.00 & 1.69 & 1.95 & 3.08 & 5.60 \\
\hline
\end{tabular}

Table 6. MRLs for the EWMA median chart based on RSS scheme when $\delta \in\{0.00,0.25,0.50,0.75,1.00,1.50,2.00\}, n=5$ and $\lambda=0.05,0.25,0.50,0.75$.

\begin{tabular}{|l|l|l|l|l|}
\hline$\delta$ & $\lambda=0.05$ & $\lambda=0.25$ & $\lambda=0.50$ & $\lambda=0.75$ \\
\hline 0.00 & 260 & 258 & 255 & 256 \\
\hline 0.25 & 71 & 123 & 162 & 191 \\
\hline 0.50 & 29 & 42 & 72 & 101 \\
\hline 0.75 & 17 & 20 & 32 & 53 \\
\hline 1.00 & 12 & 11 & 17 & 29 \\
\hline 1.50 & 8 & 6 & 7 & 10 \\
\hline 2.00 & 6 & 4 & 4 & 5 \\
\hline
\end{tabular}

\section{Performance comparison of the control chart}

In this section, the out-of-control signal detection abilities of the proposed Synthetic EWMA median chart based on RSS scheme is compared with their existing counterparts such as EWMA median chart based on RSS scheme with a list of shift sizes in the process mean.

The run-length values of the EWMA median chart based on RSS scheme are recorded in Table 4 to 6 and compared with the figures of proposed chart listed in Table 3. Same values of $n$ and $\lambda$ are considered in each comparison. A set of parameters $K$ is simulated so that the ARL of the compared chart is equal to the predefined value, which is approximately equal to 370 . 
When the figures in Table 3 and Table 6 are compared, the proposed chart outshines its opponents in terms of MRL for small to large mean shift in all the cases of $L$ except $L=1$. The proposed chart has smaller median run length performance which indicates that it can generate the out-of-control signal faster. This can be illustrated from an instance in Table 3 and Table 6 , when the $L=20, \lambda=0.25$ and $\delta=0$, the MRL of our proposed chart is 229.5 while the MRL of the EWMA median chart is 258. From the same tables, considering the other case when the $L=50, \lambda=0.50$ and $\delta=0.75$, the MRL of our proposed chart is 17 whereas the MRL of EWMA median chart is 32 .

\section{Conclusion}

The overall results establish that the run-length percentiles is a superior performance evaluation tool than the average run length (ARL) as it helps to gain a better understanding on the performance of the proposed chart. The usual practice of applying contaminated normal dataset into a mean chart is not proper as some unreliable conclusions may be acquired from the chart. In this paper, the use of Synthetic EWMA median chart as a replacement for mean chart is clearly illustrated that it can overcome the drawbacks. Besides that, the near-optimal parameters presented in Table 1 are beneficial for the practitioners on implementing the real data set into our proposed chart. Furthermore, the proposed chart is verified to have a higher sensitivity than the existing EWMA median chart in monitoring a process that involves small to large mean shift size. It is very essential for the practitioners to have a detailed knowledges of a control chart's performance features as this can intensify the practitioners' confidence on using it.

\section{References}

1. M. Best, D. Neuhauser, BMJ Qual. Saf. 15, 142 - 143 (2016)

2. J.C. Benneyan, R.C. Lloyd, P.E. Plsek, BMJ Qual. Saf. 12, 458 - 464 (2003)

3. S.M. Scariano, M.E. Calzada, Sequential Anal. 28, 54 - 68 (2009)

4. P.D. Bourke, J. Qual. Technol 23, 225 - 238 (1991)

5. K. Takahasi, K. Wakimoto, Ann. Inst. Stat. Math. 20, 1 - 31 (1968)

6. T.R. Dell, J.L. Clutter, Biometrics 28, 545 - 555 (1972)

7. R.D. Salazar, A.K. Sinha, Comunicacion Tecica 1, 1 - 97 (1997)

8. H. Muttlak, W. Al-Sabah, J. Appl. Stat. 30, 1055 - 1078 (2003)

9. A. Haq, J. Brown, E. Moltchanova, Qual. Reliab. Eng. Int. 32, 269 - 290 (2016)

10. W. Human, P. Kritzinger, S. Chakraborti, J. Appl. Stat. 38, 2071 - 2087 (2011)

11. K.L. Tan, Z.L. Chong, M.B. Khoo, W.L. Teoh, S.Y. Teh, Percentiles of the run-length distribution of the Exponentially Weighted Moving Average (EWMA) median chart, in Proceedings of the International Conference on Applied \& Industrial Mathematics and Statistics, ICoAIMS, 21 September 2017, 890, 012156 (2017)

12. P. Castagliola, P.E. Maravelakis, F.O. Figueiredo, IIE Transactions 48, 66 - 74 (2016)

13. M.B. Khoo, V.H. Wong, Z. Wu, P. Castagliola, Qual. Reliab. Eng. Int. 27, 981 - 997 (2011)

14. Q. Zhou, C. Zou, Z. Wang, W. Jiang, J. Am. Stat. Assoc. 107, 1049 - 1062 (2012) 